PROCEEDINGS OF THE

AMERICAN MATHEMATICAL SOCIETY

Volume 137, Number 10, October 2009, Pages 3283-3290

S 0002-9939(09)09917-1

Article electronically published on May 7, 2009

\title{
NONCOMMUTATIVE GEOMETRY OF ALGEBRAIC CURVES
}

\author{
IGOR V. NIKOLAEV
}

(Communicated by Varghese Mathai)

\begin{abstract}
A covariant functor from the category of generic complex algebraic curves to a category of the $A F$-algebras is constructed. The construction is based on a representation of the Teichmüller space of a curve by the measured foliations due to Douady, Hubbard, Masur and Thurston. The functor maps isomorphic algebraic curves to the stably isomorphic $A F$-algebras.
\end{abstract}

\section{INTRODUCTION}

There has been interest in the last decade in developing a dictionary between the commutative geometry of rings of polynomials in the complex projective plane and noncommutative geometry of rings of operators on a Hilbert space. The special case of elliptic curves and noncommutative tori suggests that such a dictionary exists (see [8], [9], 11], 12] et al.). In the present paper we construct a functor from the category of generic complex algebraic curves to a category of the operator algebras, known as the $A F$-algebras, which realizes such a dictionary. The functor maps isomorphic algebraic curves to the stably isomorphic (Morita equivalent) $A F$ algebras. This fact, interesting on its own, has applications e.g. in the construction of a faithful representation of the mapping class group.

Let us outline our construction. Denote by $T_{S}(g)$ the Teichmüller space of genus $g \geq 1$ with a distinguished point $S$. Let $q \in H^{0}\left(S, \Omega^{\otimes 2}\right)$ be a holomorphic quadratic differential on the Riemann surface $S$, such that all zeroes of $q$ (if any) are simple. By $\widetilde{S}$ we mean a double cover of $S$ ramified over the zeroes of $q$ and by $H_{1}^{\text {odd }}(\widetilde{S})$ the odd part of the integral homology of $\widetilde{S}$ relative to the zeroes. Note that $H_{1}^{\text {odd }}(\widetilde{S}) \cong$ $\mathbb{Z}^{n}$, where $n=6 g-6$ if $g \geq 2$ and $n=2$ if $g=1$. The fundamental result of Hubbard and Masur [7] says that $T_{S}(g) \cong \operatorname{Hom}\left(H_{1}^{o d d}(\widetilde{S}) ; \mathbb{R}\right)-\{0\}$, where 0 is the zero homomorphism.

Finally, denote by $\lambda=\left(\lambda_{1}, \ldots, \lambda_{n}\right)$ the image of a basis of $H_{1}^{\text {odd }}(\widetilde{S})$ in the real line $\mathbb{R}$, such that $\lambda_{1} \neq 0$. (Note that such an option always exists, since the zero homomorphism is excluded.) We let $\theta=\left(\theta_{1}, \ldots, \theta_{n-1}\right)$, where $\theta_{i}=\lambda_{i-1} / \lambda_{1}$. Recall that, up to a scalar multiple, the vector $(1, \theta) \in \mathbb{R}^{n}$ is the limit of a generically convergent Jacobi-Perron continued fraction [2]:

$$
\left(\begin{array}{l}
1 \\
\theta
\end{array}\right)=\lim _{k \rightarrow \infty}\left(\begin{array}{cc}
0 & 1 \\
I & b_{1}
\end{array}\right) \ldots\left(\begin{array}{cc}
0 & 1 \\
I & b_{k}
\end{array}\right)\left(\begin{array}{l}
0 \\
\mathbb{I}
\end{array}\right),
$$

Received by the editors September 5, 2008, and, in revised form, February 13, 2009

2000 Mathematics Subject Classification. Primary 14H10, 46L40, 58F10.

Key words and phrases. Complex algebraic curves, $C^{*}$-algebras.

The author was partially supported by NSERC.

(C)2009 American Mathematical Society 3283 Reverts to public domain 28 years from publication 
where $b_{i}=\left(b_{1}^{(i)}, \ldots, b_{n-1}^{(i)}\right)^{T}$ is a vector of non-negative integers, $I$ is the unit matrix and $\mathbb{I}=(0, \ldots, 0,1)^{T}$. We introduce an $A F$-algebra [4], $\mathbb{A}_{\theta}$, via the Bratteli diagram:

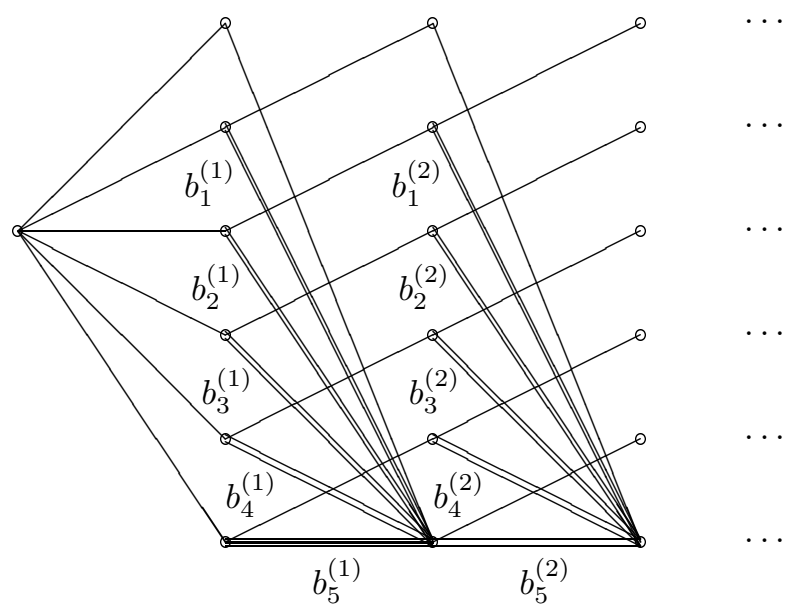

Figure 1. The Bratteli diagram of a toric $A F$-algebra $\mathbb{A}_{\theta}$ (case $g=2)$.

where the numbers $b_{j}^{(i)}$ indicate the multiplicity of edges of the graph. Let us call $\mathbb{A}_{\theta}$ a toric AF-algebra. Note that in the case $g=1$, the Jacobi-Perron fraction coincides with the regular continued fraction and $\mathbb{A}_{\theta}$ becomes the Effros-Shen $A F$ algebra of a noncommutative torus [5]. Roughly, the question addressed in this paper can be formulated as follows.

Main Problem. Suppose that $C, C^{\prime} \in T_{S}(g)$ are isomorphic complex algebraic curves of genus $g \geq 1$. Find an equivalence relation between the corresponding toric AF-algebras $\mathbb{A}_{\theta}$ and $\mathbb{A}_{\theta^{\prime}}$.

It is remarkable that the difficult question of finding an isomorphism between two algebraic curves has an amazingly simple answer in terms of operator algebras. Recall that the fundamental equivalence in noncommutative geometry is a stable isomorphism of the operator algebras rather than an isomorphism. The operator algebras $\mathbb{A}$ and $\mathbb{A}^{\prime}$ are stably isomorphic whenever $\mathbb{A} \otimes \mathcal{K}$ is isomorphic to $\mathbb{A}^{\prime} \otimes \mathcal{K}$, where $\mathcal{K}$ is the $C^{*}$-algebra of compact operators. A short answer to the main problem will be that $\mathbb{A}_{\theta}$ and $\mathbb{A}_{\theta^{\prime}}$ are stably isomorphic. Unfortunately, this beautiful fact holds only for the typical (generic) algebraic curves, which we will specify later. An obstacle in the remaining cases is a (non-generic) divergence of the Jacobi-Perron algorithm. Only in the $g=1$ case, i.e. when $\mathbb{A}_{\theta}$ is an Effros-Shen algebra, does one always have convergence.

Denote by $V$ the maximal subset of $T_{S}(g)$ such that for every complex curve $C \in V$, there exists a convergent Jacobi-Perron continued fraction. Let $F$ be the map which sends the complex algebraic curves into the toric $A F$-algebras according to the formula $C \mapsto \mathbb{A}_{\theta}$ described above. Finally, let $W$ be the image of $V$ under the mapping $F$. A summary of our results is contained in the following theorem. 
Theorem 1. The set $V$ is a generic subset of $T_{S}(g)$, and the map $F$ has the following properties:

(i) $V \cong W \times(0, \infty)$ is a trivial fiber bundle whose projection map $p: V \rightarrow W$ coincides with $F$;

(ii) $F: V \rightarrow W$ is a covariant functor which maps isomorphic complex algebraic curves $C, C^{\prime} \in V$ to stably isomorphic toric $A F$-algebras $\mathbb{A}_{\theta}, \mathbb{A}_{\theta^{\prime}} \in W$.

This paper is organized as follows. In section 1 some useful definitions are introduced, and Theorem 1 is proved in section 2 .

\section{Preliminaries}

This section is a brief review of the results necessary to prove our main theorem. For a systematic account, we refer the reader to [2, [7] and [13].

\subsection{Measured foliations and $T(g)$.}

A. A measured foliation $\mathcal{F}$ on a surface $X$ is a partition of $X$ into the singular points $x_{1}, \ldots, x_{n}$ of order $k_{1}, \ldots, k_{n}$ and regular leaves (1-dimensional submanifolds). On each open cover $U_{i}$ of $X-\left\{x_{1}, \ldots, x_{n}\right\}$ there exists a non-vanishing real-valued closed 1-form $\phi_{i}$ such that: (i) $\phi_{i}= \pm \phi_{j}$ on $U_{i} \cap U_{j}$; (ii) at each $x_{i}$ there exists a local chart $(u, v): V \rightarrow \mathbb{R}^{2}$ such that for $z=u+i v$ we have $\phi_{i}=\operatorname{Im}\left(z^{\frac{k_{i}}{2}} d z\right)$ on $V \cap U_{i}$ for some branch of $z^{\frac{k_{i}}{2}}$. The pair $\left(U_{i}, \phi_{i}\right)$ is called an atlas for the measured foliation $\mathcal{F}$. Finally, a measure $\mu$ is assigned to each segment $\left(t_{0}, t\right) \in U_{i}$ which is transverse to the leaves of $\mathcal{F}$ via the integral $\mu\left(t_{0}, t\right)=\int_{t_{0}}^{t} \phi_{i}$. The measure is invariant along the leaves of $\mathcal{F}$, hence the name.

B. Let $S$ be a Riemann surface and $q \in H^{0}\left(S, \Omega^{\otimes 2}\right)$ be a holomorphic quadratic differential on $S$. The lines $\operatorname{Re} q=0$ and $\operatorname{Im} q=0$ define a pair of measured foliations on $R$ which are transversal to each other outside the set of singular points. The set of singular points is common to both foliations and coincides with the zeroes of $q$. The above measured foliations are said to represent the vertical and horizontal trajectory structures of $q$, respectively.

C. Let $T(g)$ be the Teichmüller space of the topological surface $X$ of genus $g \geq 1$, i.e. the space of the complex structures on $X$. Consider the vector bundle $p: Q \rightarrow T(g)$ over $T(g)$ whose fiber above a point $S \in T_{g}$ is the vector space $H^{0}\left(S, \Omega^{\otimes 2}\right)$. Given non-zero $q \in Q$ above $S$, we can consider the horizontal measured foliation $\mathcal{F}_{q} \in \Phi_{X}$ of $q$, where $\Phi_{X}$ denotes the space of equivalence classes of measured foliations on $X$. If $\{0\}$ is the zero section of $Q$, the above construction defines a map $Q-\{0\} \longrightarrow \Phi_{X}$. For any $\mathcal{F} \in \Phi_{X}$, let $E_{\mathcal{F}} \subset Q-\{0\}$ be the fiber above $\mathcal{F}$. In other words, $E_{\mathcal{F}}$ is a subspace of the holomorphic quadratic forms whose horizontal trajectory structure coincides with the measured foliation $\mathcal{F}$. Note that if $\mathcal{F}$ is a measured foliation with simple zeroes (a generic case), then $E_{\mathcal{F}} \cong \mathbb{R}^{n}-0$ while $T(g) \cong \mathbb{R}^{n}$, where $n=6 g-6$ if $g \geq 2$ and $n=2$ if $g=1$.

Theorem (Hubbard-Masur [7). The restriction of $p$ to $E_{\mathcal{F}}$ defines a homeomorphism (an embedding) $h_{\mathcal{F}}: E_{\mathcal{F}} \rightarrow T(g)$.

D. The Hubbard-Masur result implies that the measured foliations parametrize the space $T(g)-\{p t\}$, where $p t=h_{\mathcal{F}}(0)$. Indeed, denote by $\mathcal{F}^{\prime}$ a vertical trajectory structure of $q$. Since $\mathcal{F}$ and $\mathcal{F}^{\prime}$ define $q$ and $\mathcal{F}=$ Const for all $q \in E_{\mathcal{F}}$, one 
gets a homeomorphism between $T(g)-\{p t\}$ and $\Phi_{X}$, where $\Phi_{X} \cong \mathbb{R}^{n}-0$ is the space of equivalence classes of the measured foliations $\mathcal{F}^{\prime}$ on $X$. Note that the above parametrization depends on the foliation $\mathcal{F}$. However, there exists a unique canonical homeomorphism $h=h_{\mathcal{F}}$ as follows. Let $S p(S)$ be the length spectrum of the Riemann surface $S$ and $S p\left(\mathcal{F}^{\prime}\right)$ the set of positive reals $\inf \mu\left(\gamma_{i}\right)$, where $\gamma_{i}$ runs over all simple closed curves which are transverse to the foliation $\mathcal{F}^{\prime}$. A canonical homeomorphism $h=h_{\mathcal{F}}: \Phi_{X} \rightarrow T(g)-\{p t\}$ is defined by the formula $S p\left(\mathcal{F}^{\prime}\right)=S p\left(h_{\mathcal{F}}\left(\mathcal{F}^{\prime}\right)\right)$ for $\mathcal{F}^{\prime} \in \Phi_{X}$. Thus, the following corollary is true.

Corollary 1. There exists a unique homeomorphism $h: \Phi_{X} \rightarrow T(g)-\{p t\}$.

E. Recall that $\Phi_{X}$ is the space of equivalence classes of measured foliations on the topological surface $X$. Following Douady and Hubbard 3 , we consider a coordinate system on $\Phi_{X}$ suitable for the proof of Theorem 1. For clarity, let us make a generic assumption that $q \in H^{0}\left(S, \Omega^{\otimes 2}\right)$ is a non-trivial holomorphic quadratic differential with only simple zeroes. We wish to construct a Riemann surface of $\sqrt{q}$ which is a double cover of $S$ with ramification over the zeroes of $q$. Such a surface, denoted by $\widetilde{S}$, is unique and has the advantage of carrying a holomorphic differential $\omega$ such that $\omega^{2}=q$. We further denote by $\pi: \widetilde{S} \rightarrow S$ the covering projection. The vector space $H^{0}(\widetilde{S}, \Omega)$ splits into the direct sum $H_{\text {even }}^{0}(\widetilde{S}, \Omega) \oplus H_{\text {odd }}^{0}(\widetilde{S}, \Omega)$ in view of the involution $\pi^{-1}$ of $\widetilde{S}$, and $H^{0}\left(S, \Omega^{\otimes 2}\right) \cong H_{\text {odd }}^{0}(\widetilde{S}, \Omega)$. Let $H_{1}^{\text {odd }}(\widetilde{S})$ be the odd part of the homology of $\widetilde{S}$ relative to the zeroes of $q$. Consider the pairing $H_{1}^{\text {odd }}(\widetilde{S}) \times H^{0}\left(S, \Omega^{\otimes 2}\right) \rightarrow \mathbb{C}$ defined by the integration $(\gamma, q) \mapsto \int_{\gamma} \omega$. We shall take the associated map $\psi_{q}: H^{0}\left(S, \Omega^{\otimes 2}\right) \rightarrow \operatorname{Hom}\left(H_{1}^{\text {odd }}(\widetilde{S}) ; \mathbb{C}\right)$ and let $h_{q}=\operatorname{Re} \psi_{q}$.

Lemma 1 (Douady-Hubbard [3]). The map $h_{q}: H^{0}\left(S, \Omega^{\otimes 2}\right) \rightarrow \operatorname{Hom}\left(H_{1}^{\text {odd }}(\widetilde{S}) ; \mathbb{R}\right)$ is an $\mathbb{R}$-isomorphism.

Since each $\mathcal{F} \in \Phi_{X}$ is the vertical foliation $\operatorname{Re} q=0$ for some $q \in H^{0}\left(S, \Omega^{\otimes 2}\right)$, the Douady-Hubbard lemma implies that $\Phi_{X} \cong \operatorname{Hom}\left(H_{1}^{\text {odd }}(\widetilde{S}) ; \mathbb{R}\right)$. By formulas for the relative homology, one finds that $H_{1}^{\text {odd }}(\widetilde{S}) \cong \mathbb{Z}^{n}$, where $n=6 g-6$ if $g \geq 2$ and $n=2$ if $g=1$. Finally, each $h \in \operatorname{Hom}\left(\mathbb{Z}^{n} ; \mathbb{R}\right)$ is given by the reals $\lambda_{1}=h\left(e_{1}\right), \ldots, \lambda_{n}=h\left(e_{n}\right)$, where $\left(e_{1}, \ldots, e_{n}\right)$ is a basis in $\mathbb{Z}^{n}$. The numbers $\left(\lambda_{1}, \ldots, \lambda_{n}\right)$ are the coordinates in the space $\Phi_{X}$ and, in view of Corollary 1 , in the Teichmüller space $T(g)$.

\subsection{The Jacobi-Perron continued fraction.}

A. Let $a_{1}, a_{2} \in \mathbb{N}$ be such that $a_{2} \leq a_{1}$. Recall that the greatest common divisor of $a_{1}$ and $a_{2}, G C D\left(a_{1}, a_{2}\right)$, can be determined from the Euclidean algorithm:

$$
\left\{\begin{array}{cc}
a_{1} & =a_{2} b_{1}+r_{3} \\
a_{2} & =r_{3} b_{2}+r_{4} \\
r_{3} & =r_{4} b_{3}+r_{5} \\
\vdots & \\
r_{k-3} & =r_{k-2} b_{k-1}+r_{k-1} \\
r_{k-2} & =r_{k-1} b_{k},
\end{array}\right.
$$


where $b_{i} \in \mathbb{N}$ and $G C D\left(a_{1}, a_{2}\right)=r_{k-1}$. The Euclidean algorithm can be written as the regular continued fraction

$$
\theta=\frac{a_{1}}{a_{2}}=b_{1}+\frac{1}{b_{2}+\frac{1}{+\cdots+\frac{1}{b_{k}}}}=\left[b_{1}, \ldots b_{k}\right]
$$

If $a_{1}$ and $a_{2}$ are non-commensurable in the sense that $\theta \in \mathbb{R}-\mathbb{Q}$, then the Euclidean algorithm never stops and $\theta=\left[b_{1}, b_{2}, \ldots\right]$. Note that the regular continued fraction can be written in the matrix form

$$
\left(\begin{array}{l}
1 \\
\theta
\end{array}\right)=\lim _{k \rightarrow \infty}\left(\begin{array}{cc}
0 & 1 \\
1 & b_{1}
\end{array}\right) \ldots\left(\begin{array}{cc}
0 & 1 \\
1 & b_{k}
\end{array}\right)\left(\begin{array}{l}
0 \\
1
\end{array}\right) .
$$

B. The Jacobi-Perron algorithm and connected (multidimensional) continued fraction generalizes the Euclidean algorithm to the case $G C D\left(a_{1}, \ldots, a_{n}\right)$ when $n \geq 2$. Specifically, let $\lambda=\left(\lambda_{1}, \ldots, \lambda_{n}\right)$ with $\lambda_{i} \in \mathbb{R}-\mathbb{Q}$ and $\theta_{i-1}=\frac{\lambda_{i}}{\lambda_{1}}$ for $1 \leq i \leq n$. The continued fraction

$$
\left(\begin{array}{c}
1 \\
\theta_{1} \\
\vdots \\
\theta_{n-1}
\end{array}\right)=\lim _{k \rightarrow \infty}\left(\begin{array}{ccccc}
0 & 0 & \ldots & 0 & 1 \\
1 & 0 & \ldots & 0 & b_{1}^{(1)} \\
\vdots & \vdots & & \vdots & \vdots \\
0 & 0 & \ldots & 1 & b_{n-1}^{(1)}
\end{array}\right) \ldots\left(\begin{array}{ccccc}
0 & 0 & \ldots & 0 & 1 \\
1 & 0 & \ldots & 0 & b_{1}^{(k)} \\
\vdots & \vdots & & \vdots & \vdots \\
0 & 0 & \ldots & 1 & b_{n-1}^{(k)}
\end{array}\right)\left(\begin{array}{c}
0 \\
0 \\
\vdots \\
1
\end{array}\right),
$$

where $b_{i}^{(j)} \in \mathbb{N} \cup\{0\}$, is called the Jacobi-Perron algorithm (JPA). Unlike the regular continued fraction algorithm, the JPA may diverge for certain vectors $\lambda \in \mathbb{R}^{n}$. However, for points in a generic subset of $\mathbb{R}^{n}$, the JPA converges. We characterize such a set in the next paragraph.

C. The convergence of the JPA algorithm can be characterized in terms of the measured foliations. Let $\mathcal{F} \in \Phi_{X}$ be a measured foliation on the surface $X$ of genus $g \geq 1$. Recall that $\mathcal{F}$ is called uniquely ergodic if every invariant measure of $\mathcal{F}$ is a multiple of the Lebesgue measure. By the Masur-Veech theorem, there exists a generic subset $V \subset \Phi_{X}$ such that each $\mathcal{F} \in V$ is uniquely ergodic [10, 14. We let $\lambda=\left(\lambda_{1}, \ldots, \lambda_{n}\right)$ be the vector with coordinates $\lambda_{i}=\mu\left(\gamma_{i}\right)$, where $\gamma_{i} \in H_{1}^{\text {odd }}(\widetilde{S})$; see $\S 1$ 1. E. By an abuse of notation, we shall say that $\lambda \in V$. By a duality between the measured foliations and the interval exchange transformations [10], there is the following characterization of convergence of the JPA.

Lemma 2 (Bauer 1]). The JPA converges if and only if $\lambda \in V \subset \mathbb{R}^{n}$.

\section{Proof of Theorem 1}

Let us outline the proof. We consider the following categories: (i) generic complex algebraic curves $\mathcal{V}=V \subset T(g)$, (ii) pseudo-lattices $\mathcal{P} L$, (iii) projective pseudolattices $\mathcal{P} P L$, and (iv) category $\mathcal{W}$ of the toric $A F$-algebras. First, we show that $\mathcal{V} \cong \mathcal{P} L$ are equivalent categories, so that isomorphic complex algebraic curves $C, C^{\prime} \in \mathcal{V}$ map to isomorphic pseudo-lattices $P L, P L^{\prime} \in \mathcal{P} L$. Next, a non-injective functor $F: \mathcal{P} L \rightarrow \mathcal{P} P L$ is constructed; $F$ maps isomorphic pseudo-lattices to isomorphic projective pseudo-lattices and $\operatorname{Ker} F \cong(0, \infty)$. Finally, it is shown that 
a subcategory $U \subseteq \mathcal{P} P L$ and $\mathcal{W}$ are the equivalent categories. In other words, we have the following diagram:

$$
\mathcal{V} \stackrel{\alpha}{\longrightarrow} \mathcal{P} L \stackrel{\mathrm{F}}{\longrightarrow} U \stackrel{\beta}{\longrightarrow} \mathcal{W}
$$

where $\alpha$ is an injective map, $\beta$ is a bijection and $\operatorname{Ker} F \cong(0, \infty)$.

Category $\mathcal{V}$. Let $\operatorname{Mod} X$ be the mapping class group of the surface $X$. A complex algebraic curve is a triple $(X, C, j)$ where $X$ is a topological surface of genus $g \geq 1$, $j: X \rightarrow C$ is a complex (conformal) parametrization of $X$, and $C$ is a Riemann surface. A morphism of complex algebraic curves $(X, C, j) \rightarrow\left(X, C^{\prime}, j^{\prime}\right)$ is the identity $j \circ \psi=\varphi \circ j^{\prime}$, where $\varphi \in \operatorname{Mod} X$ is a diffeomorphism of $X$ and $\psi$ is an isomorphism of Riemann surfaces. A category $\mathcal{V}$ of generic complex algebraic curves consists of $O b(\mathcal{V})$, the complex algebraic curves $C \in V \subset T_{S}(g)$, and morphisms $H\left(C, C^{\prime}\right)$ between $C, C^{\prime} \in O b(\mathcal{V})$ which coincide with the morphisms specified above. For any $C, C^{\prime}, C^{\prime \prime} \in O b(\mathcal{V})$ and any morphisms $\varphi^{\prime}: C \rightarrow C^{\prime}$ and $\varphi^{\prime \prime}: C^{\prime} \rightarrow C^{\prime \prime}$, a morphism $\phi: C \rightarrow C^{\prime \prime}$ is the composite of $\varphi^{\prime}$ and $\varphi^{\prime \prime}$, which we write as $\phi=\varphi^{\prime \prime} \varphi^{\prime}$. The identity morphism, $1_{C}$, is a morphism $H(C, C)$.

Category $\mathcal{P} L$. A pseudo-lattic 1 (of rank $n$ ) is a triple $\left(\Lambda, \mathbb{R}, j\right.$ ) where $\Lambda \cong \mathbb{Z}^{n}$ and $j: \Lambda \rightarrow \mathbb{R}$ is a homomorphism. A morphism of pseudo-lattices $(\Lambda, \mathbb{R}, j) \rightarrow\left(\Lambda, \mathbb{R}, j^{\prime}\right)$ is the identity $j \circ \psi=\varphi \circ j^{\prime}$, where $\varphi$ is a group homomorphism and $\psi$ is an inclusion map, i.e. $j^{\prime}\left(\Lambda^{\prime}\right) \subseteq j(\Lambda)$. Any isomorphism class of a pseudo-lattice contains a representative given by $j: \mathbb{Z}^{n} \rightarrow \mathbb{R}$ such that $j(1,0, \ldots, 0)=\lambda_{1}, \quad j(0,1, \ldots, 0)=$ $\lambda_{2}, \quad \ldots, \quad j(0,0, \ldots, 1)=\lambda_{n}$, where $\lambda_{1}, \lambda_{2}, \ldots, \lambda_{n}$ are positive reals. The pseudolattices of rank $n$ make up a category, which we denote by $\mathcal{P} L_{n}$.

Lemma 3 (Basic lemma). Let $g \geq 2$ (respectively $g=1$ ) and $n=6 g-6$ (respectively $n=2)$. There exists an injective covariant functor $\alpha: \mathcal{V} \rightarrow \mathcal{P} L_{n}$ which maps isomorphic complex algebraic curves $C, C^{\prime} \in \mathcal{V}$ to the isomorphic pseudo-lattices $P L, P L^{\prime} \in \mathcal{P} L_{n}$.

Proof. Let $\alpha: T(g)-\{p t\} \rightarrow \operatorname{Hom}\left(H_{1}^{\text {odd }}(\widetilde{S}) ; \mathbb{R}\right)-\{0\}$ be the map constructed in $\S 1.1$. By the corollary of the Hubbard-Masur theorem, $\alpha$ is a homeomorphism. In particular, $\alpha$ is an injective map.

Let us find the image $\alpha(\varphi) \in \operatorname{Mor}(\mathcal{P} L)$ of $\varphi \in \operatorname{Mor}(\mathcal{V})$. Let $\varphi \in \operatorname{Mod} X$ be a diffeomorphism of $X$, and let $\widetilde{X} \rightarrow X$ be the ramified double cover of $X$ as explained in $\S 1.1$.E. We denote by $\widetilde{\varphi}$ the induced mapping on $\widetilde{X}$. Note that $\widetilde{\varphi}$ is a diffeomorphism of $\widetilde{X}$ modulo the covering involution $\mathbb{Z}_{2}$. Denote by $\widetilde{\varphi}^{*}$ the action of $\widetilde{\varphi}$ on $H_{1}^{\text {odd }}(\widetilde{X}) \cong \mathbb{Z}^{n}$. Since $\widetilde{\varphi} \bmod \mathbb{Z}_{2}$ is a diffeomorphism of $\widetilde{X}$, we have $\widetilde{\varphi}^{*} \in G L_{n}(\mathbb{Z})$. Thus, $\alpha(\varphi)=\widetilde{\varphi}^{*} \in \operatorname{Mor}(\mathcal{P} L)$.

Let us show that $\alpha$ is a functor. Indeed, let $C, C^{\prime} \in \mathcal{V}$ be isomorphic complex algebraic curves such that $C^{\prime}=\varphi(C)$ for a $\varphi \in \operatorname{Mod} X$. Let $a_{i j}$ be the elements of matrix $\widetilde{\varphi}^{*} \in G L_{n}(\mathbb{Z})$. Recall that $\lambda_{i}=\int_{\gamma_{i}} \phi$ for a closed 1-form $\phi=\operatorname{Re} \omega$ and $\gamma_{i} \in H_{1}^{\text {odd }}(\widetilde{X})$. Then $\gamma_{j}=\sum_{i=1}^{n} a_{i j} \gamma_{i}, j=1, \ldots, n$, are the elements of a new basis in $H_{1}^{\text {odd }}(\widetilde{X})$. By the integration rules,

$$
\lambda_{j}^{\prime}=\int_{\gamma_{j}} \phi=\int_{\sum a_{i j} \gamma_{i}} \phi=\sum_{i=1}^{n} a_{i j} \lambda_{i} .
$$

\footnotetext{
${ }^{1}$ See [9].
} 
Finally, let $j(\Lambda)=\mathbb{Z} \lambda_{1}+\cdots+\mathbb{Z} \lambda_{n}$ and $j^{\prime}(\Lambda)=\mathbb{Z} \lambda_{1}^{\prime}+\cdots+\mathbb{Z} \lambda_{n}^{\prime}$. Since $\lambda_{j}^{\prime}=$ $\sum_{i=1}^{n} a_{i j} \lambda_{i}$ and $\left(a_{i j}\right) \in G L_{n}(\mathbb{Z})$, we conclude that $j(\Lambda)=j^{\prime}(\Lambda) \subset \mathbb{R}$. In other words, the pseudo-lattices $(\Lambda, \mathbb{R}, j)$ and $\left(\Lambda, \mathbb{R}, j^{\prime}\right)$ are isomorphic. Hence, $\alpha: \mathcal{V} \rightarrow$ $\mathcal{P} L$ maps isomorphic complex algebraic curves to the isomorphic pseudo-lattices; i.e. $\alpha$ is a functor.

Finally, let us show that $\alpha$ is a covariant functor. Indeed, let $\varphi_{1}, \varphi_{2} \in \operatorname{Mor}(\mathcal{V})$. Then $\alpha\left(\varphi_{1} \varphi_{2}\right)=\left(\widetilde{\varphi_{1} \varphi_{2}}\right)^{*}=\widetilde{\varphi}_{1}^{*} \widetilde{\varphi}_{2}^{*}=\alpha\left(\varphi_{1}\right) \alpha\left(\varphi_{2}\right)$. Lemma 3 follows.

Category $\mathcal{P} P L$. A projective pseudo-lattice (of rank $n$ ) is a triple $(\Lambda, \mathbb{R}, j$ ) where $\Lambda \cong \mathbb{Z}^{n}$ and $j: \Lambda \rightarrow \mathbb{R}$ is a homomorphism. A morphism of projective pseudolattices $(\Lambda, \mathbb{C}, j) \rightarrow\left(\Lambda, \mathbb{R}, j^{\prime}\right)$ is the identity $j \circ \psi=\varphi \circ j^{\prime}$, where $\varphi$ is a group homomorphism and $\psi$ is an $\mathbb{R}$-linear map. (Notice that, unlike the case of pseudolattices, $\psi$ is a scaling map as opposed to an inclusion map. This allows the two pseudo-lattices to be projectively equivalent while being distinct in the category $\mathcal{P} L_{n}$.) It is not hard to see that any isomorphism class of a projective pseudolattice contains a representative given by $j: \mathbb{Z}^{n} \rightarrow \mathbb{R}$ such that $j(1,0, \ldots, 0)=$ $1, \quad j(0,1, \ldots, 0)=\theta_{1}, \ldots, \quad j(0,0, \ldots, 1)=\theta_{n-1}$, where $\theta_{i}$ are positive reals. The projective pseudo-lattices of rank $n$ make up a category, which we denote by $\mathcal{P P} L_{n}$.

Category $W$. Finally, the toric $A F$-algebras $\mathbb{A}_{\theta}$, modulo the stable isomorphism between them, make up a category, which we shall denote by $W_{n}$.

Lemma 4. Let $U_{n} \subseteq \mathcal{P} P L_{n}$ be a subcategory consisting of the projective pseudolattices $P P L=P P L\left(1, \theta_{1}, \ldots, \theta_{n-1}\right)$ for which the Jacobi-Perron fraction of the vector $\left(1, \theta_{1}, \ldots, \theta_{n-1}\right)$ converges to the vector. Define a map $\beta: U_{n} \rightarrow W_{n}$ by the formula PPL $\left(1, \theta_{1}, \ldots, \theta_{n-1}\right) \mapsto \mathbb{A}_{\theta}$, where $\theta=\left(\theta_{1}, \ldots, \theta_{n-1}\right)$. Then $\beta$ is a bijective functor which maps isomorphic projective pseudo-lattices to the stably isomorphic toric AF-algebras.

Proof. It is evident that $\beta$ is injective and surjective. Let us show that $\beta$ is a functor. Indeed, according to 4, Corollary 4.7, every totally ordered abelian group of rank $n$ has the form $\mathbb{Z}+\theta_{1} \mathbb{Z}+\cdots+\mathbb{Z} \theta_{n-1}$. The latter is a projective pseudolattice $P P L$ from the category $U_{n}$. On the other hand, by the Elliott theorem [6], the $P P L$ defines a stable isomorphism class of the toric $A F$-algebra $\mathbb{A}_{\theta} \in W_{n}$. Therefore, $\beta$ maps isomorphic projective pseudo-lattices (from the set $U_{n}$ ) to the stably isomorphic toric $A F$-algebras, and vice versa. Lemma 4 follows.

Let $P L\left(\lambda_{1}, \lambda_{2}, \ldots, \lambda_{n}\right) \in \mathcal{P} L_{n}$ and $P P L\left(1, \theta_{1}, \ldots, \theta_{n-1}\right) \in \mathcal{P} P L_{n}$. To finish the proof of Theorem 1, it remains to prove the following lemma.

Lemma 5. Let $F: \mathcal{P} L_{n} \rightarrow \mathcal{P} P L_{n}$ be a map given by $P L\left(\lambda_{1}, \lambda_{2}, \ldots, \lambda_{n}\right) \mapsto$ $\operatorname{PPL}\left(1, \frac{\lambda_{2}}{\lambda_{1}}, \ldots, \frac{\lambda_{n}}{\lambda_{1}}\right)$. Then $\operatorname{Ker} F=(0, \infty)$ and $F$ is a functor which maps isomorphic pseudo-lattices to isomorphic projective pseudo-lattices.

Proof. Indeed, $F$ can be thought of as a map from $\mathbb{R}^{n}$ to $\mathbb{R} P^{n}$. Hence $\operatorname{Ker} F=$ $\left\{\lambda_{1}: \lambda_{1}>0\right\} \cong(0, \infty)$. The second part of the lemma is evident.

Setting $n=6 g-6$ (respectively, $n=2$ ) for $g \geq 2$ (respectively, $g=1$ ) in Lemmas 3 [5, one gets the conclusion of Theorem 1 .

\section{ACKNOWLEDGMENT}

I am grateful to Yu. I. Manin for helpful advice. 


\section{REFERENCES}

[1] M. Bauer, A characterization of uniquely ergodic interval exchange maps in terms of the Jacobi-Perron algorithm, Bol. Soc. Bras. Mat. 27 (1996), 109-128. MR1418928 (98a:58100)

[2] L. Bernstein, The Jacobi-Perron Algorithm, Its Theory and Applications, Lect. Notes in Math., 207, Springer, 1971. MR0285478 (44:2696)

[3] A. Douady and J. Hubbard, On the density of Strebel differentials, Inventiones Math. 30 (1975), 175-179. MR0396936(53:796)

[4] E. G. Effros, Dimensions and $C^{*}$-Algebras, Conf. Board of the Math. Sciences Regional Conference Series in Math., No. 46, Amer. Math. Soc., 1981. MR623762 (84k:46042)

[5] E. G. Effros and C. L. Shen, Approximately finite $C^{*}$-algebras and continued fractions, Indiana Univ. Math. J. 29 (1980), 191-204. MR563206 (81g:46076)

[6] G. A. Elliott, On the classification of inductive limits of sequences of semisimple finitedimensional algebras, J. Algebra 38 (1976), 29-44. MR0397420 (53:1279)

[7] J. Hubbard and H. Masur, Quadratic differentials and foliations, Acta Math. 142 (1979), 221-274. MR523212 (80h:30047)

[8] M. Kontsevich, XI Solomon Lefschetz Memorial Lecture Series: Hodge structures in noncommutative geometry (Notes by Ernesto Lupercio), in Contemp. Mathematics, 462, Amer. Math. Soc., 2008. MR2444365

[9] Yu. I. Manin, Real multiplication and noncommutative geometry, in "Legacy of Niels Hendrik Abel", 685-727, Springer, 2004. MR2077591(2006e:11077)

[10] H. Masur, Interval exchange transformations and measured foliations, Ann. of Math. (2) 115 (1982), no. 1, 169-200. MR644018 (83e:28012)

[11] A. Polishchuk and A. Schwarz, Categories of holomorphic vector bundles on noncommutative two-tori, Commun. Math. Phys. 236 (2003), 135-159. MR.1977884 (2004k:58011)

[12] Y. Soibelman and V. Vologodsky, Noncommutative compactifications and elliptic curves, Int. Math. Res. Not. (2003), 1549-1569. MR1976601 (2004i:14017)

[13] W. P. Thurston, On the geometry and dynamics of diffeomorphisms of surfaces, Bull. Amer. Math. Soc. 19 (1988), 417-431. MR956596 (89k:57023)

[14] W. A. Veech, Gauss measures for transformations on the space of interval exchange maps, Ann. of Math. (2) 115 (1982), no. 1, 201-242. MR644019 (83g:28036b)

The Fields Institute for Mathematical Sciences, Toronto, Ontario, M5T 3J1, Canada Current address: 101-315 Holmwood Avenue, Ottawa, Ontario, K1S 2R2, Canada

E-mail address: igor.v.nikolaev@gmail.com 